\title{
ON THE OBLIQUE BOUNDARY PROBLEM WITH A STOCHASTIC INHOMOGENEITY
}

\author{
THOMAS RASKOP AND MARTIN GROTHAUS
}

\begin{abstract}
We analyze the regular oblique boundary problem for the Poisson equation on a $C^{1,1}$-domain with stochastic inhomogeneities. At first we investigate the deterministic problem. Since our assumptions on the inhomogeneities and coefficients are very weak, already in order to formulate the problem we have to work out properties of functions from Sobolev spaces on submanifolds. An further analysis of Sobolev spaces on submanifolds together with the Lax-Milgram lemma enables us to prove an existence and uniqueness result for the weak solution to the oblique boundary problem under very weak assumptions on coefficients and inhomogeneities. Then we define the spaces of stochastic functions with help of the tensor product. These spaces enable us to extend the deterministic formulation to the stochastic setting. Under as weak assumptions as in the deterministic case we are able to prove the existence and uniqueness of a stochastic weak solution to the regular oblique boundary problem for the Poisson equation. Our studies are motivated by problems from geodesy and through concrete examples we show the applicability of our results. Finally a Ritz-Galerkin approximation is provided. This can be used to compute the stochastic weak solution numerically.
\end{abstract}

\section{INTRODUCTION}

In this paper we treat the regular oblique boundary problem for the Poisson equation

$$
\begin{array}{rll}
\Delta u=f & \text { in } & \Gamma, \\
(\underline{a} \cdot \nabla u)+b u=\tilde{g} & \text { on } & \partial \Gamma, \\
|(\underline{a} \cdot \underline{\nu})| \geq \gamma>0 & \text { on } & \partial \Gamma
\end{array}
$$

on a bounded $C^{1,1}$-domain $\Gamma \subset \mathbb{R}^{n}$, where $\underline{\nu}$ is the outward unit normal vector on $\partial \Gamma$. The coefficients $\underline{a}$, b and the inhomogeneities $\mathrm{f}, \tilde{g}$ are elements of Sobolev spaces defined on $\Gamma$ or $\partial \Gamma$, respectively. Assuming smooth enough coefficients and inhomogeneities it can be transformed into the equivalent form:

$$
\begin{aligned}
& \Delta u=f \quad \text { in } \quad \Gamma, \\
& \frac{\partial u}{\partial \underline{\nu}}+\left(\underline{\alpha} \cdot \nabla_{\partial \Gamma} u\right)+\beta u=g \quad \text { on } \quad \partial \Gamma,
\end{aligned}
$$

Date: June 29, 2009.

Key words and phrases. Poisson equation, oblique boundary problem, stochastic inhomogeneities, Sobolev spaces on submanifolds, geodesy, Ritz-Galerkin approximation.

2000 Mathematics Subject Classification. 35J05, 35J25, 35R05, 35R60, 46E35, 60G60.

We thank a very conscientious referee for a careful reading of the manuscript and making very useful comments and suggestions. 


$$
(\underline{\alpha} \cdot \underline{\nu})=0 \quad \text { on } \quad \partial \Gamma .
$$

Now we are able to derive a weak formulation. Under the assumptions

$$
\begin{aligned}
\underline{\alpha} & \in H^{1, \infty}(\partial \Gamma ; T(\partial \Gamma)), \\
\beta & \in L^{\infty}(\partial \Gamma), \\
\operatorname{ess}_{\inf _{\partial \Gamma}\left(\beta-\frac{1}{2} \operatorname{div}_{\partial \Gamma}(\underline{\alpha})\right)} & 0, \\
f & \in\left(H^{1,2}(\Gamma)\right)^{\prime}, \\
g & \in H^{-\frac{1}{2}, 2}(\partial \Gamma)
\end{aligned}
$$

we can ensure the existence and uniqueness of a weak solution in $H^{1,2}(\Gamma)$. It is well known that one can ensure the existence of a classical solution $u \in C^{2, h}(\bar{\Gamma})(h \in[0,1])$ of $(1.1)$ - (1.3) for all

$$
\begin{aligned}
& f \in C^{0, h}(\bar{\Gamma}), \\
& \tilde{g} \in C^{1, h}(\partial \Gamma)
\end{aligned}
$$

under the following conditions, see [GiTr01, Theorem 6.31]:

$$
\begin{aligned}
\underline{a} & \in C^{1, h}\left(\partial \Gamma ; \mathbb{R}^{n}\right), \\
b & \in C^{1, h}(\partial \Gamma), \\
\Gamma & \mathrm{a} C^{2, h}-\text { domain, } \\
(\underline{a} \cdot \underline{\nu}) b & >0 \text { on } \partial \Gamma .
\end{aligned}
$$

We generalize our weak concept in such a way that the inhomogeneities and consequently the solution additionally are stochastic:

$$
\begin{aligned}
\Delta u(x, \omega) & =f(x, \omega) \quad \text { for all } x \in \Gamma, \text { P-a.a. } \omega \in \Omega, \\
(\underline{a} \cdot \nabla u(x, \omega))+b u(x, \omega) & =g(x, \omega) \quad \text { for all } x \in \partial \Gamma, \text { P-a.a. } \omega \in \Omega, \\
|(\underline{a} \cdot \underline{\nu})| \geq \gamma & >0 \quad \text { on } \partial \Gamma .
\end{aligned}
$$

Since we are using tensor products and the Lax-Milgram lemma in our approach to include stochastic inhomogeneities we need a Hilbert space. Hence $C^{2, h}(\bar{\Gamma})$ is as a Banach space not suitable for us. We use the tensor product to define the function spaces:

$$
\begin{aligned}
\left(H^{1,2}(\Gamma)\right)_{\Omega}^{\prime} & :=L^{2}(\Omega, P) \otimes\left(H^{1,2}(\Gamma)\right)^{\prime}, \\
H_{\Omega}^{-\frac{1}{2}, 2}(\partial \Gamma) & :=L^{2}(\Omega, P) \otimes H^{-\frac{1}{2}, 2}(\partial \Gamma), \\
H_{\Omega}^{1,2}(\Gamma) & :=L^{2}(\Omega, P) \otimes H^{1,2}(\Gamma) .
\end{aligned}
$$

These enable us to derive a weak formulation for the stochastic regular oblique boundary problem for the Poisson equation. Now one can adapt the techniques from the deterministic case to the probabilistic one to get existence and uniqueness of a stochastic weak solution in $H_{\Omega}^{1,2}(\Gamma)$ for:

$$
\begin{aligned}
& f \in\left(H^{1,2}(\Gamma)\right)_{\Omega}^{\prime}, \\
& g \in H_{\Omega}^{-\frac{1}{2}, 2}(\partial \Gamma) .
\end{aligned}
$$


One also can translate the main theorems of this paper to the Neumann and Dirichlet boundary problems where one also has a weak formulation and a corresponding weak solution in the Hilbert spaces $H^{1,2}(\Gamma)$ for the Neumann problem and $H_{0}^{1,2}(\Gamma)$ for the Dirichlet problem respectively, see [Alt02, Chapter 4]. The Neumann problem can be considered as a special case of the oblique boundary problem. For the Dirichlet problem the technique of the stochastic extension and the main theorems can be translated easily with slight modifications of the proofs. Finally we show how to apply the developed theory in praxis. For example we can treat Gaussian inhomogeneities as used in [FBa04] and [FrMa02]. Additionally we provide the Ritz-Galerkin method to approximate the stochastic weak solution by a sequence $\left(u_{n}\right)_{n \in \mathbb{N}}$, where $u_{n}$ is the stochastic weak solution in a finite dimensional subspace $U_{n} \subset H_{\Omega}^{1,2}(\Gamma)$ with $U_{n} \subset U_{n+1}$ such that $\overline{\bigcup_{n \in \mathbb{N}} U_{n}}=$ $H_{\Omega}^{1,2}(\Gamma)$. This offers a possibility to compute the solution by numerical simulations.

The analysis of our problem is motivated by models from geodesy. Here the problem is defined in terms of an outer problem, i.e.

$$
\begin{array}{rll}
\Delta u^{*}=f^{*} & \text { in } & \mathbb{R}^{n} \backslash \Gamma, \\
\left(\underline{a}^{*} \cdot \nabla u^{*}\right)+b^{*} u^{*}=g^{*} & \text { on } & \partial \Gamma, \\
\left|\left(\underline{a}^{*} \cdot \underline{\nu}\right)\right| \geq \gamma^{*}>0 & \text { on } & \partial \Gamma,
\end{array}
$$

where $\Gamma$ is at least a $C^{2}$-domain, see [FrMi04]. But by a Kelvin transformation it can be transformed into an inner problem, see e.g. [RoSa03], which is investigated in our paper. The stochastic inhomogeneities can be used to implement a random source of error occurring in measurements. Moreover our contribution generalizes the result obtained in [RoSa02].

This paper is organized as follows: First we work out some properties of functions from Sobolev spaces, see Section 2. Then after we have developed the formulation in Section 3.1, we prove the existence and uniqueness of a weak solution in Section 3.2, see Theorem 3.7. This result is basic for the stochastic one, see Theorem 4.4. An important ingredient is the Lax-Milgram lemma. Then we extend the spaces as well as the formulation of the problem to the stochastic case in Section 4.1. Main tool here is the tensor product. In Section 4.2 the existence and uniqueness result from the deterministic case is translated to the probabilistic setting. In Section 5.1 - Section 5.3 we give examples to show that the results are really applicable, see [FBa04] or [FrMa02]. Like already announced above we also derive a Ritz-Galerkin approximation for our stochastic weak solution of (1.4) (1.6) in the last section, see Section 6.

The main progress achieved in this paper can be summarized by the following:

- The existence and uniqueness of a weak solution of the deterministic oblique boundary problem for the Poisson equation for $C^{1,1}$-domains under very weak assumptions on coefficients and inhomogeneities is proved, see Theorem 3.7.

- These results are translated into the stochastic case and existence and uniqueness of a stochastic weak solution is proved, see Theorem 4.4.

- Examples for applications to concrete stochastic inhomogeneities as used in [FBa04] and [FrMa02] are given.

- A Ritz-Galerkin approximation of the solution is provided. This is of interest for numerical simulations. 
Thomas Raskop, Mathematics Department, University of Kaiserslautern, P.O.Box 3049, 67653 Kaiserslautern, Germany.

Email: thomasraskop@gmx.de

Martin Grothaus, Mathematics Department, University of Kaiserslautern, P.O.Box 3049, 67653 Kaiserslautern, Germany; Bibos, Bielefeld University, 33615 Bielefeld, Germany and SFB 611, IAM, University of Bonn, 53115 Bonn, Germany.

Email: grothaus@mathematik.uni-kl.de, URL: http://www.mathematik.uni-kl.de/ grothaus/ 